ANÁLISE REGIONAL DAS MESORREGIOES DO ESTADO DO PARANÁ NO FINAL DO SÉCULO XX

JANDIR FERRERA DE LIMA, LUCIR REINALDO ALVES, MOACIR Piffer e Carlos Alberto Piacenti

CUSTO NA DÍVIDA PÚBLICA INTERNA DA REDUÇĀO DA VULNERABILIDADE EXTERNA BRASILEIRA ATRAVESS DO AUMENTO DAS RESERVAS INTERNACIONAIS

ROBERTO MEURER

DETERMINAČÃO DE UM MODELO DE PREVISÃO UNIVARIADO PARA PREÇOS DE LEITE PAGOS AOS PRODUTORES EM SANTA CATARINA

ARLei LUiz Fachinello e Mirian Rumenos Piedade Bacch!

VIABILIDADE DE ESTRATÉGIAS DE HEDCE COM CONTRATOS FUTUROS DE BOI GORDO NO BRASIL DIANA de MEDEIROS BAPTISTA E DANILO ROLIM DIAS DE AGUIAR

ATAQUES ESPECULATIVOS E CRISES CAMBIAIS NA ARGENTINA E NO BRASIL: UMA ANÁLISE COMPARATIVA KELLEN FrAGa DA SIIVA E FERnANDO FERRARI FILHO

A TEORIA DOS FUNDOS DE EMPRÉSTIMOS: UM ESTUDO DOS MODELOS AGREGADOS NEOCLÁSSICO E KEYNESIANO

ALAIN HERSCOVICI

CRIME ECONÔMICO NO PARANÁ: UM ESTUDO DE CASO SALETE POLONIA BoriLLI E PERY Francisco AsSIS SHIKIDA

APLICAÇĀO DA NOVA ECONOMIA INSTITUCIONAL AO AMBIENTE PORTUÁRIO: ANÁLISE DOS CUSTOS DE TRANSAÇĀO NO PORTO DE SANTOS

CinTIA RETZ LUCCI, ALCINDO FERNANDES GONÇALVES E

ROBERTO FAVA SCARE

REDUÇÃO DE MANDATOS LEGISLATIVOS: O DEBATE ADORMECIDO

Francisco josé de Queiroz PINHeiro, Charles lima de ALMEIDA E TITO BELCHIOR SILVA MOREIRA

ANÁLISE ECONÔMICA E AMBIENTAL DE SISTEMAS DE TERMINACCÃO DE SUINNOS COM A APLICACCÃO DOS CONUUNTOS FUZZY

JULIO EDUARDo ROHENKOHL, ORLANDO MARTINELLI E MARCOS ALVES DOS REYS

RESENHA: THE GLOBAL EVOLUTION OF INDUSTRIAL RELATIONS EVENTS, IDEAS AND THE IIRA

CARLOS HENRIQUE HoRN
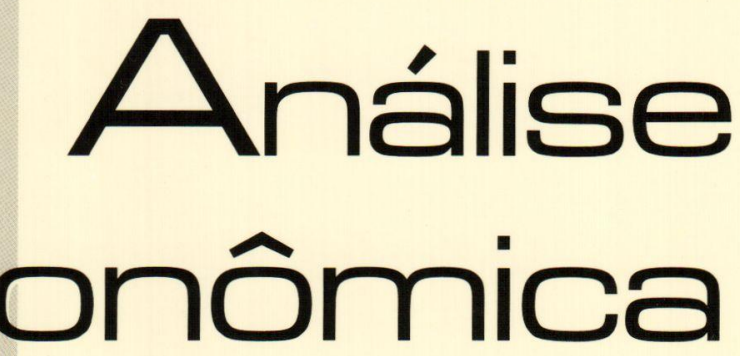


\section{A Revista Análise Econômica agradece a colaboração dos pareceristas dos números 45 e 46 , abaixo relacionados}

Abraham Benzaquen Sicsu

Adelar Fochezalto

Ademar Ribeiro Romeiro

Ademir Clemente

Alexandre Stamford da Silva

Ana Lucia Kassouf

Andre Luis Rossi de Oliveira

Andre Tosi Furtado

Andrea Sales Soares de Azevedo Melo

Angela Antonia Kageyama

Antonio Wilson Ferreira Menezes

Armando João Dalla Costa

Bernardo Mueller

Carlos Frederico Leao Rocha

Claudio Roberto Fóffano Vasconcelos

Cláudio Diissey Shikida

Clesio Lourenco Xavier

Dullio de Avila Berni

Eliezer Martins Diniz

Emerson Fernandes Marçal

Eugenio Lagemann

Fernando Ferrari Fitho

Francisco Casimiro Filho

Franklin Leon Peres Serrano

Frederico Gonzaga Jayme Jr.

Geraldo Edmundo Silva Jr.

Helder Ferreira de Mendonça
Helder Queiroz Pinto Junior Izabel Cristina Takitane

Joaquim José Martins Guilhoto

Joilson Dias

Jose Gabriel Porcile Meirelles

José Rubens Damas Garlipp

Julio César de Oliveira

Lovois de Andrade Miguel

Marcelo Savino Portugal

Marcio Holland de Brito

Marco Aurelio Crocco Afonso

Marcos Costa Holanda

Mônica Viegas Andrade

Paulo Dabdab Waquil

Paulo Sergio Fracalanza

Pedro Bandeira

Pedro Valentim Marques

Pery Francisco Assis Shikida

Renato Leite Marcondes

Roberto Camps Moraes

Ronald Otto Hilbrech

Ronaldo de Albuquerque e Arraes

Ronaldo Seroa da Motta

Thompson Almeida Andrade

Tito Belchior Silva Moreira

Valmor Marchetti

Vladimir Kuhl Teles 


\title{
A teoria dos fundos de empréstimos: um estudo dos modelos agregados Neoclássico e Keynesiano
}

\author{
Alain Herscovici*
}

Resumo: A crítica que Keynes faz da teoria clássica dos fundos de empréstimos revela as incompatibilidades entre o modelo heurístico apresentado na Teoria Geral e aquele da teoria neoclássica padrão. No âmbito deste trabalho, analisarei essas incompatibilidades a partir (a) dos mecanismos que permitem determinar a taxa de juros, a poupança e a preferência pela liquidez (b) da estrutura agregada dos dois modelos. Mostrarei igualmente, como, a partir da refutação da existência de um mercado do trabalho e do capital, esta análise permite refutar a lei de Say.

Palavras-chave: taxas de juros, Lei de Say, preferência pela liquidez

Código JEL: E12 (Keynes; Keynesian; Post-Keynesian) - B41 (Economic Methodology)

Abstract: Keynes's critique of the classical theory of loanable funds reveals the incompatibilities between the heuristic model developed in The General Theory and the converse one from the standard neoclassical theory. In this paper these incompatibilities are analyzed from the viewpoint of (a) the mechanisms that determine the interest rate, savings and the liquidity preference, as well as (b) the aggregate structure of both models. Also, it will be shown how, from the refutation of the existence of a market for labor and capital, such analysis allows the refutation of Say's Law.

Keywords: interest rate, Say's Law, liquidity preference

JEL Classification: E12 (Keynes; Keynesian; Post-Keynesian) - B41 (Economic Methodology)

A crítica radical que Keynes faz da teoria dos fundos de empréstimos (TFE) revela as incompatibilidades metodológicas e epistemológicas que existem entre a estrutura agregada do modelo neoclássico e do modelo keynesiano, da maneira como ela é apresentada na Teoria Geral. ${ }^{1}$

\footnotetext{
* Doutor em Economia pelas Universidades de Paris I Panthéon-Sorbonne e de Amiens, Coordenador do Grupo de Estudo em Macroeconomia (GREM) do Departamento de Economia da UFES, Pesquisador do Conselho Nacional de Desenvolvimento Científico e Tecnológico (CNPq), Coordenador e Professor do Mestrado (e-mail: alhersco.vix@terra.com.br).

Recebido em dezembro de 2005. Aceito em maio de 2006.

${ }^{1}$ Para simplificar a notação, colocarei TG no corpo do texto para John Maynard Keynes, A teoria geral do emprego, do juro e da moeda, Atlas, São Paulo, 1990
} 
O objetivo principal deste trabalho consiste em mostrar como essa crítica se implementa em dois tempos: num primeiro tempo, Keynes faz uma crítica da TFE e mostra que é impossível determinar a taxa de juros a partir desta teoria. Num segundo tempo, sua concepção da natureza e das modalidades de determinação da taxa de juros lhe permite construir um modelo agregado no qual as relações de causalidade são totalmente diferentes daquelas apresentadas pela escola neoclássica.

Numa primeira parte, ressaltarei os fundamentos da crítica que Keynes faz à TFE; numa segunda parte, mostrarei como esta crítica implica estruturas agregadas diferentes no que diz respeito ao modelo neoclássico e ao modelo exposto na TG.

\section{Keynes e a TFE: os fundamentos da crítica}

\subsection{A crítica de Keynes}

No capítulo 14 da TG, Keynes ressalta os limites, ou seja, os problemas de coerência interna da teoria clássica no que diz respeito à TFE. Por escola clássica, Keynes designa os economistas, segundo os quais a poupança é sempre igual ao investimento (TG, p. 145). Esta abordagem "clássica" deixa voluntariamente de lado o problema da poupança forçada e da polêmica entre Keynes e Robertson. Por outro lado, esta dimensão estática (HERSCOVICI, 2002) constitui o fundamento desta crítica.

A crítica feita por Keynes é diretamente ligada às modalidades de determinação da taxa de juros e, conseqüentemente, à natureza da poupança. Para os clássicos, a demanda por fundos (ou por recursos) é assimilada ao investimento; simetricamente, a oferta de fundos corresponde à poupança (TG, p. 143). A taxa "natural" é a taxa que corresponde à igualação entre a demanda e a oferta de fundos, para uma situação de pleno emprego. Este raciocínio parte dos seguintes pressupostos:

i) Em nível agregado, a taxa de juros é determinada endogenamente pela demanda e pela oferta de fundos.

ii) Na tradição marshalliana, o investimento, ou seja, a demanda por uma determinada quantidade de capital, corresponde à demanda por fundos; a taxa de juros é o preço pago pelo uso do capital em qualquer mercado (TG, p. 143). Ela é concebida como a taxa de aluguel do capital e é obrigatoriamente igual à taxa de lucro.

iii) Nesta perspectiva, por definição, a poupança financia o investimento. 
A taxa de juros representa o prêmio pela espera, pelo fato de renunciar ao consumo presente; finalmente, esta taxa de juros natural representa a taxa que permite igualar investimento e poupança a um nivel que corresponde ao pleno emprego, no sentido de não haver desemprego involuntário. No âmbito deste trabalho, mostrarei como, e por que, Keynes refuta esses resultados.

Para Keynes, a demanda e a oferta de fundos determinam a taxa de juros a partir do momento que a renda é constante (TG, p. 145). Apenas nessas condições, é possível determinar endogenamente a taxa de juros a partir da TFE; para um nível de renda dado, a poupança é determinada pela taxa de juros. No entanto, o deslocamento da curva de demanda por fundos (o qual corresponderia a uma variação da eficiência marginal do capital, na TG) é incompatível com o fato de a renda permanecer constante (TG, pp. 145 e 146). A partir do momento que existe o efeito multiplicador, a qualquer variação do investimento corresponde uma variação da renda.

$\mathrm{Na}$ teoria clássica, a taxa de juros é a recompensa pela espera, no que diz respeito ao consumo. Na tradição de Mills e de Jevons, o ser racional seria dotado de uma preferência pelo consumo presente: a taxa de juros seria a recompensa para ele adiar este consumo. É interessante já observar que, a partir de tal perspectiva, a poupança é uma escolha intertemporal de consumo: nos modelos neoclássicos de crescimento oriundos do modelo canônico de Solow (1956), a poupança representa um aumento do consumo futuro. De fato, se a renda atual permanece constante, a um aumento da poupança atual corresponde uma diminuição do consumo atual, um aumento do investimento e, conseqüentemente, um aumento do consumo futuro.

No seio da construção neoclássica, a TFE cumpre uma dupla função: (a) é uma outra forma de expressar a lei de Say (PETRI, 1998); o fato de a poupança financiar o investimento significa, em nível agregado, que toda a renda distribuída nas atividades de produção corresponde, direta ou indiretamente, aos gastos efetuados na totalidade da economia (b) ela parte do pressuposto que existe uma relação monotônica entre a taxa de juros e o investimento. Tal relação permite explicar as modalidades de ajustamento para a posição de equilíbrio e remete diretamente à problemática da natureza do capital ${ }^{2}$ (HERSCOVICI, 2004 (b)).

2 Para uma apresentação sintética da controvérsia do capital, ver Pasinetti (1997) e Cohen e Harcourt (2003). 
De fato, no modelo da TG, Investimento e Poupança são determinados exogenamente (TG, p. 148) e de maneira independente: $\mathrm{o}$ in vestimento depende da diferença entre a eficiência marginal do capital e a taxa de juros, e a poupança da renda. As divergências entre os dois modelos (aquele exposto na TG e o modelo clássico) podem ser formalizadas da seguinte maneira:

$$
\begin{aligned}
& \mathrm{I}=\varphi_{1}[\mathrm{e}-\mathrm{r}] \\
& \operatorname{com} \varphi_{1}^{-}>0 \\
& \mathrm{Y}=\varphi_{2}(\mathrm{I}) \\
& \operatorname{com} \varphi_{2}^{-}>0 \\
& \mathrm{~S}=\varphi_{3}(\mathrm{Y}) \\
& \operatorname{com} \varphi_{3}^{-}>0 .
\end{aligned}
$$

(e representa a eficiência marginal do capital, $r$ a taxa de juros, I e $S$, respectivamente, o investimento e a poupanç, a e $\mathrm{Y}$ a renda).

São três equações ${ }^{3}$ e quatro incógnitas, e, r, I e Y; não é possível resolver este sistema. Em outras palavras, como afirma Keynes, não é possivel determinar simultaneamente, a partir deste sistema, a taxa de juros e a renda.

A solução clássica consiste em exogenizar a renda; neste caso, a renda é determinada na esfera real, a partir de uma função de produção agregada bem comportada, na qual existe uma substituabilidade perfeita dos fatores de produção. A taxa de juros é determinada pela TFE; na tradição clássica, a taxa de juros é igual à taxa de lucro e à produtividade marginal do capital. A neutralidade da moeda é dupla: por um lado, a moeda não afeta a renda e, por outro lado, está tendo uma determinação real (e não monetária) da taxa de juros.

No sistema keynesiano, ao contrário, existe uma determinação exógena da taxa de juros (VERCELLI, 1991, p. 184, PASINETTI, 1997, p. 212). As implicações são as seguintes:

i) A TFE deixa de ser explicativa, na medida em que ela não permite determinar a taxa de juros de equilibrio;

ii) A exogenidade da taxa de juros se explica a partir de sua determinação monetária. Por determinação monetária, entende-se suas relações com a incerteza e com sua função de reserva de valor.

${ }^{3} \mathrm{Na}$ medida em que $S$ depende de $\mathrm{Y}$, é possível considerar, apenas, as equações (I) e (3); neste caso, são duas equações e três incógnitas: $e, r$ e Y. Da mesma maneira, não é possível resolvèr o sistema. 
iii) A partir deste sistema simples de equaçōes, é possivel deduzir que a renda é determinada pela taxa de juros, ou seja, por uma variável monetária, no sentido precedentemente definido; isto é sinônimo de não neutralidade da moeda.

Os mecanismos de ajustamento que permitem passar de uma posição de desequilíbrio para a posição de equilíbrio são igualmente diferentes. No âmbito da análise de Keynes na TG, o ajustamento se implementa a partir de uma variação da renda, pelo viés do efeito multiplicador: a variação do investimento gera a variação da renda necessária para igualar, ex-post, poupança e investimento globais.

$\mathrm{Na}$ tradição clássica, ao contrário, o ajustamento se dá a partir de uma variação da taxa de juros, este movimento explicando a convergência sistemática para a taxa natural de juros. É igualmente importante observar que, na TFE, investimento e poupança são determinados a partir da mesma variável - a taxa de juros -, e que esta taxa de juros é, ela mesma, determinada pela poupança e pelo investimento.

$$
\begin{aligned}
& \Delta \mathrm{I}=\mathrm{f}_{1}(\Delta \mathrm{r}) \\
& \operatorname{com} \mathrm{f}_{1}^{\prime}<0 \\
& \Delta \mathrm{P}=\mathrm{f}_{2}(\Delta \mathrm{r}) \\
& \operatorname{com} \mathrm{f}_{2}^{\prime}>0 \\
& \Delta \mathrm{r}=\mathrm{f}_{3}(\mathrm{I}-\mathrm{P}) \\
& \operatorname{com} \mathrm{f}_{3}^{\prime}>0 \\
& \Leftrightarrow \Delta \mathrm{I}=\left[\mathrm{f}_{1} \mathrm{f}_{3}(\mathrm{I}-\mathrm{P})\right]=\Phi_{1}(\mathrm{I}-\mathrm{P}) \\
& \operatorname{com} \Phi_{1}^{\prime}<0 \\
& \Leftrightarrow \Delta \mathrm{P}=\left[\mathrm{f}_{2} \cdot \mathrm{f}_{3^{\prime}} .(\mathrm{I}-\mathrm{P})\right]=\Phi_{2}(\mathrm{I}-\mathrm{P}) \\
& \operatorname{com} \Phi_{2}^{\prime}>0
\end{aligned}
$$

Este sistema composto ressalta o fato de que o sistema converge, automaticamente, para a posição de equilíbrio estável representada pela taxa natural de juros. Há um aumento do investimento (6) corresponde um aumento de r; este aumento gera, num segundo momento, uma queda do investimento (4) e um aumento da poupança (5), até alcançar a nova posição de equilíbrio. É importante observar que a curva de oferta de fundos representa a poupança quando o nível da renda é constante; conseqüentemente, o aumento da poupança se traduz obrigatoriamente por uma queda do consumo. 


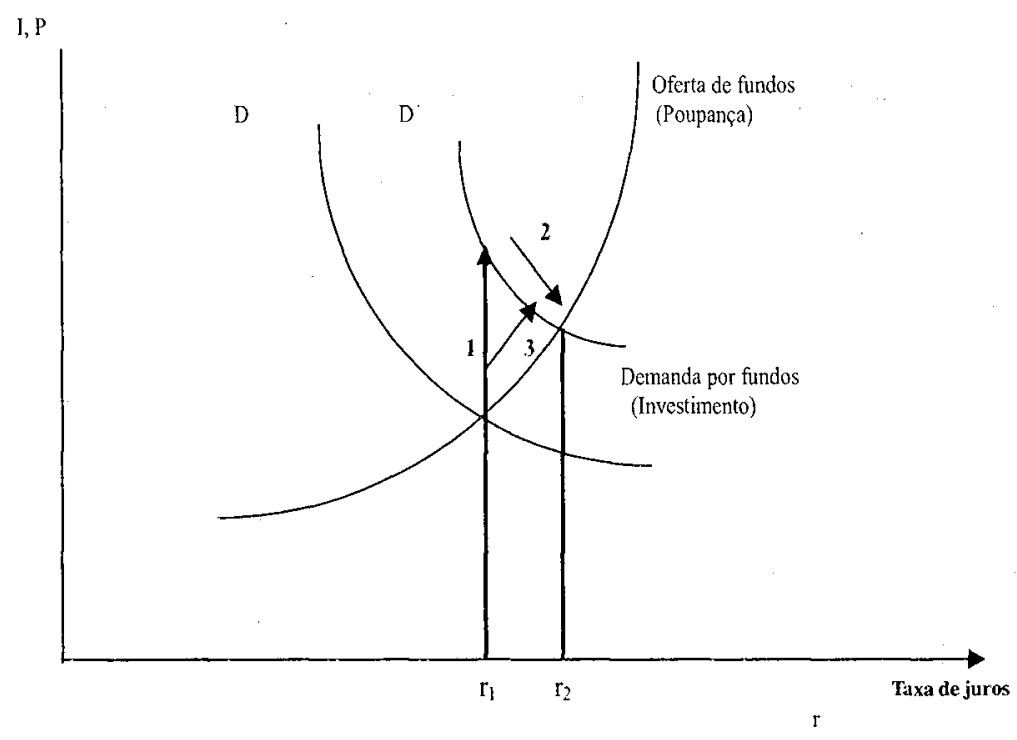

Figura 1. A TFE e a convergência para a taxa natural de juros

\section{Os determinantes da taxa de juros e a preferência pela liquidez}

No esquema keynesiano, é o nível da taxa de juros que, em última instância, determina as condições do equilíbrio, ou seja, o ponto no qual o fluxo de investimento vai parar. É necessário identificar vários níveis de problemática: quais são os elementos que determinam a taxa de juros? Quais são as características do equilíbrio e em que medida ele é diferente do equilíbrio neoclássico?

A taxa de juros não equilibra poupança e investimento no nível que corresponde ao pleno emprego, mas permite igualar "o desejo de manter a riqueza em forma líquida" com a oferta de moeda exógena determinada, por hipótese, pelo Banco Central (TG, p. 37). A demanda por moeda, ou seja, a preferência pela liquidez, depende de três motivos: transação e precaução (LI), os quais dependem da renda, e especulação (L2) que depende, de maneira inversa, da taxa de juros.

Como é possivel definir o conceito de preferência pela liquidez (PL) em Keynes? A ambigüidade do pensamento de Keynes provém do fato que ele apenas considera a demanda de moeda por motivo de especulação como fonte de incerteza. A demanda de moeda por motivo de especulação se explica da seguinte maneira: se os especuladores esperam um aumento da taxa de juros, eles esperam uma queda dos valores das obrigações. Conseqüentemente, a demanda de moeda por motivo de especulação, demanda relativa ao período atual, vai diminuir. $\mathrm{Na}$ medida em que esses agentes estão prevendo uma diminuição do valor 
do título financeiro, no período $t+1$, eles só vão comprar títulos em $t+1$ e não em $t$. Os agentes têm que escolher entre a moeda e a compra de títulos. Ern $t$, eles vão escolher a moeda, o que significa que está tendo um aumento da PL; trata-se da escolha dos ativos no período $t$.

O raciocínio de Keynes, no que diz respeito à demanda por moeda, limita a incerteza às expectativas relativas às variações da taxa de juros: neste caso, a preferência pela liquidez é, ela também, limitada às atividades que se relacionam com a especulação. Enquanto a demanda de moeda que depende diretamente da renda implica a neutralidade da moeda, apenas a demanda de moeda por motivo de especulação explicaria sua relação com a incerteza; as expectativas relativas às evoluções da taxa de juros seriam, assim, a principal fonte de incerteza. Acredito que o universo construído por Keynes caracteriza-se pela incerteza (DAVIDSON, 1996), mais especificamente no que diz respeito à decisão de investimento, e que esta incerteza se relaciona com o conjunto das decisões dos agentes; por isto, a demanda de moeda por motivo de precaução está diretamente ligada à incerteza que cerca qualquer tipo de decisão econômica. Quanto maior esta incerteza, ou seja, a avaliação subjetiva realizada pelos diferentes agentes (HERSCOVICI ALAIN, 2004 (a)), maior a preferência pela liquidez (KAHN, 1972). Assim, a preferência pela liquidez não pode ser limitada às atividades de especulação; o investimento, por exemplo, é uma decisão irreversível para o empresário, decisão tomada na base de expectativas de longo prazo incertas. A incerteza e a preferência pela liquidez que lhe correspondem têm que contemplar este tipo de situação; de um modo geral, a partir da retenção de moeda ociosa, a PL representa um meio de se prevenir contra a incerteza, que ela seja ligada às variações das taxas de juros ou de qualquer outra variável econômica. ${ }^{4}$ Assim, a preferência pela liquidez não pode ser limitada às atividades que se relacionam com a especulação.

Se chamamos $L_{1}$ a demanda de moeda que depende da renda, $\mathrm{L}_{2}$ a demanda de moeda ligada à incerteza relativa à especulação e $\mathrm{L}_{3}$ a demanda de moeda ligada à incerteza "em geral", a demanda total de moeda pode ser determinada pela seguinte equação:

$$
\mathrm{L}=\mathrm{L}_{1}(\mathrm{Y})+\mathrm{L}_{2}(\mathrm{r})+\mathrm{L}_{3}\left(\Omega_{2}\right)
$$

$\left(\Omega_{2}\right.$ representa a incerteza que não está ligada às atividades especulativas)

$$
\mathrm{L}_{3}^{\prime}>0, \mathrm{~L}_{2}^{\prime}<0, \mathrm{~L}_{1}>0
$$

A ambigüidade provém do fato que existem dois tipos de PL: a PL ligada à especulação, ou seja, às expectativas relativas às variações da taxa de juros, $\left(\mathrm{PL}_{1}\right)$, e a incerteza que cerca o conjunto das decisöes econômicas, mais especificamente a decisão de investimento $\left(\mathrm{PL}_{2}\right)$. Assim, em função de (9), a PL total é igual a:

${ }^{4}$ A este respeito, Vercelli (1999, p. 24) fala em demanda especulativa por moeda, a qual depende do maior prêmio por liquidez apresentada pela retenção de moeda. 


$$
\begin{aligned}
& \mathrm{PL}=\varphi_{1}(\mathrm{Y})+\varphi_{2}\left(\mathrm{PL}_{1}\right)+\varphi_{3}\left(\mathrm{PL}_{2}\right) \\
& \varphi_{1}^{\prime}>0, \varphi_{2}^{\prime}>0 \mathrm{e} \varphi_{3}^{\prime}>0 .
\end{aligned}
$$

Esta preferência pela liquidez global se explica a partir de três componentes: apenas um desses componentes $(\varphi 1)$ é determinado pela esfera real, ou seja, pelo nível da renda. Os dois outros se relacionam com ativos não reprodutíveis, sejam os títulos financeiros, seja a moeda. A PL assim definida se explica por uma escolha dos ativos: ativos reais, financeiros ou monetários. Nesta perspectiva, a taxa de juros é determinada a partir da preferência pela liquidez assim definida; neste sentido, a moeda, em função de sua relação intrínseca com a incerteza, determina a "esfera real". E por meio desta concepção da moeda que é possivel falar em economia monetária de produção.

No que diz respeito ao mercado monetário, a taxa de juros é determinada exogenamente pela preferência pela liquidez (PL), a partir do seguinte mecanismo: a preferência pela liquidez determina a demanda por moeda, a qual explica, num segundo momento, as variaçōes da taxa de juros necessárias para igualar, novamente, demanda e oferta por moeda (TG, p. 137). Existe assim uma determinação monetária da taxa de juros, esta sendo explicada, em última instância, pela PL.

No modelo neoclássico, ao contrário, a taxa de juros determina a demanda por moeda e é concebida como o custo de oportunidade de se reter moeda (FRIEDMAN, 1974, p. 25); quanto maior este custo, menor a demanda por moeda. Por outro lado, o fato de a taxa de juros ser igual, na posição de equilíbrio, à produtividade marginal do capital, a qual é igual à taxa de lucro, no modelo neoclássico, não permite deduzir, como o faz Jevons, que a taxa de juros é determinada pela produtividade marginal do capital (DENIS, HENRI, op. cit., p. 499). Encontramos, novamente, a crítica de Keynes à TFE:

i) taxa de juros e produtividade marginal do capital (eficiência marginal do capital, na TG) são determinadas de maneira independente: a taxa de juros depende da incerteza e da PL, a eficiência marginal do capital, das expectativas de longo prazo dos empresários.

ii) a igualdade entre taxa de juros e eficiência marginal do capital caracteriza apenas a posição de equilibrio, na qual o retomo previsto do último investimento realizado é igual à taxa de juros. A taxa de juros é determinada exogenamente e sem nenhuma relação com a eficiência marginal do capital $^{5}$ (PETRI, op. cit., 1998), a partir de mecanismos institucionais e/ou da avaliação que os agentes fazem da incerteza.

\footnotetext{
${ }^{5}$ Concordo assim plenamente com Pasinetti quando ele afirma que "se a taxa de juros determina o investimento, a recíproca não é verificada" (PASINETTl, op. cit., p. 209
} 
A análise de Keynes na TG nega a relação de causalidade neoclássica; esta negação ressalta a determinaçẫo monetária da taxa de juros e o fato de a moeda determinar o investimento, ou seja, a esfera real. De fato, a causalidade do modelo neoclássico é a seguinte: o investimento e a poupança determinam a taxa de juros (a partir da TFE), a qual determina a demanda por moeda. Ao contrário, na TG, a PL determina a demanda por moeda e, num segundo momento, as variações da taxa de juros que permitem igualar demanda e oferta por moeda. Assim, na teoria neoclássica, a demanda por moeda é determinada pela taxa de juros, enquanto na TG, a taxa de juros é determinada pela demanda por moeda.

\section{' 2 A estrutura dos modelos neoclássico e keynesiano}

\subsection{Os principais mecanismos}

A taxa de juros $r$ depende da PL e da oferta de moeda.

$$
\begin{aligned}
& r=\mathrm{f}_{2}(\mathrm{PL}-\mathrm{Om}) \\
& \operatorname{comf}_{2}^{\prime}>0 .
\end{aligned}
$$

A equação (11) ressalta os seguintes resultados:

i) quanto maior a incerteza, maior será a retenção de moeda para se prevenir contra esta incerteza, e maior a taxa de juros;

ii) se a PL varia na mesma proporção que a oferta de moeda, uma variação da oferta de moeda não se traduzirá por uma variação da taxa de juros. A este respeito, Keynes afirma que "Embora seja de esperar que, ceteris paribus, um aumento na quantidade de moeda reduz a taxa de juros, isto não ocorrerá se a preferência do público pela liquidez aumentar mais que a quantidade de moeda" (TG, p. 141).

A eficiência marginal do capital $e$ depende das expectativas de longo prazo dos empresários e da demanda por bens de capital, ou seja, do investimento:

$e=f_{3}(e L P-I)$

(eLP: expectativas de longo prazo, I investimento)

A partir do conceito de preço de oferta (TG, p. 115 e 116), Keynes chega às seguintes conclusões: (a) quanto maiores as expectativas de longo prazo, para um mesmo preço de oferta, maior e; (b) existe uma relação inversa entre o volume total do investimento e a eficiência marginal do capital total; Keynes mostra que, quando o investimento aumenta, esta eficiência diminui (TG, p. 115 e 116). ${ }^{6}$

${ }^{6}$ Para uma análise detalhada deste mecanismo, ver Herscovici (2004 (b)). 
$O$ investimento depende da diferença entre $e$ e $r$, o que permite escrever, a partir de (11) e (12):

$$
\Delta \mathrm{I}=\left[\mathrm{f}_{3}(\mathrm{eL} \cdot \mathrm{P}-\mathrm{I})-\mathrm{f}_{2}(\mathrm{PL}-\mathrm{Om})\right]
$$

A equação (13) permite formular os seguintes resultados:

i) quanto maiores as expectativas de longo prazo, maiores as variações do investimento.

ii) quanto maior a oferta de moeda, maiores as variações do investimento.

iii) quanto maior o nível do investimento, menores suas variações futuras. Finalmente, existe uma relação positiva entre, de um lado, as variações do investimento e, de outro, as variações da renda e do produto.

i) Em função dos problemas relativos à mensuração do capital, Keynes vai medir o produto a partir "das horas de trabalho pagas e aplicadas no equipamento existente tanto para satisfazer os consumidores como para produzir novo equipamento de capital" (TG, p. 51); ele valoriza o produto total a partir da quantidade total de trabalho gasta na produção.

ii) Por outro lado, quando Keynes constrói as funções de demanda e de oferta agregadas, ele relaciona o produto esperado com determinada quantidade de trabalho (TG, capítulo 3);

iii) Finalmente, o multiplicador de investimento ressalta a correlação positiva entre as variações do investimento e a variação da renda e, conseqüentemente, do nível de emprego.

O multiplicador se expressa pela seguinte relação:

$\Delta \mathrm{Y}=(\mathrm{I} / \mathrm{s}) \cdot \Delta \mathrm{I}=\mathrm{M} \cdot \Delta \mathrm{I}$

( $s$ representa a propensão marginal a poupar)

o que implica:

$\Delta \mathrm{Y}=\mathrm{M} .\left[\mathrm{f}_{3}(\mathrm{eLP}-\mathrm{I})-\mathrm{f}_{2}(\mathrm{PL}-\mathrm{Om})\right]$

À medida que as variações do produto se traduzem por variações correspondentes do nível de emprego:

$$
\begin{aligned}
& \Delta \mathrm{N}=\alpha \cdot \mathrm{M} \cdot\left[\mathrm{f}_{3}(\mathrm{eLP}-\mathrm{I})-\mathrm{f}_{2}(\mathrm{PL}-\mathrm{Om})\right] \\
& (\alpha \text { é uma constante positiva })
\end{aligned}
$$

As equações (15) e (16) permitem formular os seguintes resultados:

i) Existe uma correlação positiva entre o nível da renda (e o nível de emprego), as expectativas de longo prazo, a oferta de moeda e a propensão marginal a consumir. Um aumento da renda e do nível de emprego correspondente pode ser obtido a partir de uma modificação das expectativas de longo prazo elaboradas pelos empresários (CARDIM, 1999) e/ou por qualquer medida que possa aumentar a propensão marginal a consumir da coletividade. Duas observações fazem-se necessárias: uma política econômica cujo objetivo consiste em diminuir os salários reais 
não permite aumentar sistematicamente a renda nem o nivel de emprego. A diminuição do salário real se traduz por uma queda do consumo, o que se traduz por uma diminuição das expectativas de longo prazo e por uma diminuição da propensão marginal a consumir da coletividade. Por outro lado, sobre certas condições (o aumento de Om sendo superior ao aumento de $\mathrm{PL}$ ), um aumento da massa monetária correspondente provoca um aumento da renda e do nível de emprego.

ii) Existe uma correlação negativa entre o nível da renda, o nível do investimento e a preferência pela liquidez. A primeira relação expressa o fato de o sistema produzir endogenamente flutuaçôes, ou mais precisamente, alternância entre fases de expansão e recessão (HERSCOVICI ALAIN, 2005); a segunda expressa o fato que, quando a incerteza aumenta, os agentes retêm mais moeda, o que se traduz por uma diminuição das expectativas de longo prazo relativas ao investimento e por uma transferência da demanda dos ativos reprodutíveis para ativos cuja elasticidade de produção é praticamente nula (DAVIDSON, 1999, KREGEL,1980. ); neste caso, o emprego perdido na produção de bens de capital reprodutivel não é compensado pela "produção" de ativos não reprodutiveis.

Estes resultados são incompatíveis com aqueles da teoria neoclássica, seja na versão walrasiana, monetarista, novo-keynesiana ou novo-clássica: isto ressalta as incompatibilidades no que diz respeito à estrutura geral dos modelos heurísticos (VERCELLI, 1991, op. cit.).

\subsection{A estrutura agregada dos modelos}

O modelo agregado neoclássico se caracteriza por uma hierarquia entre os diferentes mercados: no curto prazo, o equilíbrio se realiza, em um primeiro momento, no mercado do trabalho. O salário real, determinado a partir da oferta e da demanda por trabalho, é tal que corresponde a uma situação de pleno emprego. Num segundo momento, o nível da renda se determina em função da quantidade de trabalho que corresponde ao equilíbrio no mercado do trabalho. A teoria quantitativa da moeda ressalta o fato que um aumento da massa monetária se traduz por um aumento do nível geral dos preços, não modifica os preços relativos e não afeta a renda real. A moeda é neutra, tanto no longo quanto no curto prazo, pelo fato de não afetar a renda nem o nível de emprego. A demanda de moeda é unicamente determinada pela renda e pela velocidade de circulação da moeda. Enfim, no caso que nos interessa, o mercado dos fundos de empréstimos é totalmente desconectado dos mercados do trabalho e dos bens e serviços (SIMONSEN, p. 34, A FROIS, p. 124). Em outras palavras, o equilibrio no mercado do trabalho e no mercado dos bens e serviços é independente do equilíbrio realizado no mercado dos fundos de empréstimos e no mercado da moeda. Esta estrutura 
corresponde à validação da lei de Say. Nesta perspectiva, à uma diminuição dos salários reais corresponde um aumento da quantidade de trabalho empregado e um aumento da renda.

Por outro lado, é interessante observar que os autores neoclássicos que tentaram reformular as modalidades de determinação da taxa de juros chegaram a resultados incompatíveis com os resultados neoclássicos. Marshall, por exemplo, não consegue compatibilizar sua teoria monetária das flutuações de curto prazo com a existência de uma taxa de juros real que corresponderia ao equilíbrio de longo prazo (BRIDEL, 1987, p. 37 e seguintes): não consegue explicar o processo de ajustamento da taxa de juros de mercado para o valor da taxa de juros real, nem compatibilizar esta análise das flutuações de curto prazo com sua teoria de longo prazo do investimento (Idem, p. 43).

A estrutura do modelo da TG é totalmente diferente:

i) $\mathrm{O}$ fato de não haver hierarquização dos diferentes mercados significa que a moeda não é neutra: ela influencia, no curto e no longo prazo, a renda e o nível de emprego.

ii) conforme ressaltei neste trabalho, a taxa de juros não é determinada no mercado do capital a partir de um jogo de oferta e de demanda. Por outro lado, não existe nenhum mecanismo econômico que determine sistematicamente a taxa de juros que corresponde ao pleno emprego: ao contrário, a multiplicidade dos equilibrios possiveis mostra que a renda (de equilíbrio) varia em função das expectativas de longo prazo dos empresários e da avaliação que os agentes fazem da incerteza, ou seja, da preferência pela liquidez do público.

iii) Finalmente, o investimento "real" depende diretamente da taxa de juros, esta taxa sendo determinada, no mercado monetário, a partir da incerteza avaliada pelos agentes econômicos; assim, a cada mudança desta avaliação corresponde uma mudança na taxa de juros, no nivel do investimento, do emprego e da renda. Contrariamente ao modelo neoclássico, as modalidades da taxa de juros determinam o nível da renda.

Pàra Keynes, não éxistem mercado do trabalho nem mercado do capital (ROTHEIM, 1988). No que diz respeito ao primeiro ponto, Keynes refuta o segundo postulado da teoria clássica, ou seja, o fato de a oferta de trabalho ser determinada pela igualação entre o salário real e a desutilidade do trabalho (TG, p. capítulo 2), partindo do fato que os trabalhadores negociam seus salários em termos nominais e não reais (CHICK, 1993, p. 14, VERCELLI, 1991, p. 179). No que diz respeito ao segundo ponto, Keynes rejeita o próprio conceito de mercado do capital, pelas seguintes razões: (a) Keynes era consciente do problema relativo à mensuração do capital (HERSCOVICI, 2004 (b)): por isto, não é possível medir, em valor, uma determinada quantidade de capital, 
tanto do lado da demanda quanto da oferta (PASINETTI, op. cit.). Por esta razão, Keynes resolve medir a variação da renda a partir da quantidade de trabalho empregada, e não em valor. (b) Keynes refuta a determinação clássica da taxa de juros a partir de um mercado do capital, ou seja, a partir da TFE.

\section{Observações finais}

Em conclusão, é possivel afirmar que a crítica de Keynes à TFE ressalta a diferença e as incompatibilidades com a matriz neoclássica, no que diz respeito às modalidades de determinação das variáveischave como a taxa de juros e a poupança. A determinação monetária da taxa de juros salienta a interdependência entre o real e o monetário, ou seja, o fato de as economias capitalistas serem economias monetárias de produção. De fato, esta crítica permite ressaltar as incompatibilidades metodológicas entre a estrutura do modelo agregado apresentado na TG e aquela da teoria neoclássica padrão.

Por outro lado, esta crítica se relaciona diretamente, a meu ver, com o fato de não haver, na construção apresentada na TG, um mercado do capital, no sentido neoclássico da palavra: esta convergência com a análise neo-ricardiana permite questionar os fundamentos dos modelos neo-clássicos à medida que não é mais possível conceber uma posição de crescimento equilibrado que corresponda a uma quantidade constante, em valor, de capital, nem um processo de ajustamento para esta posição (HARRIS, 1978, capítulo 9).

\section{Referências}

ABRAHAM-FROIS, Gilbert. Dynamique Economique, 7ème édition, Dalloz, Paris, 1991. BRIDEL, Pascal. Cambridge Monetary thought. The development of saving-invesment analysis from Marshall to Keynes, St Martin's Press, New York, 1987.

CARDIM DE CARVALHO, Fernando J. Políticas Econômicas par Economias Monetárias, in Macroeconomia moderna. Keynes e a Economia Contemporânea, G.T. Lima. J. Sicsú. L.F. de Paulo, orgs, Editora Campus, Rio de janeiro, 1999.

CHICK, Victoria. Macroeconomia após Keynes. Um reexame da Teoria Geral, Forense Universitária, Rio de Janeiro, 1993.

COHEN, Avi J.; HARCOURT G.C. Whatever Happened to the Cambridge Capital Theory Controversies?, in Journal of Economic Perspectives - Volume 17, Winter 2003.

DAVIDSON, Paul. Reality and economic theory, Joumal of Post Keynesian Economics/ Summer 1996.

Herscovici, A. A teoria dos fundos de empréstimos... 
DAVIDSON, Paul. Colocando as Evidências em Ordem: Macroeconomia de Keynes versus Velhos e Novos Keynesianos, in Macroeconomia moderna. Keynes e a Economia Contemporânea, G.T. Lima. J. Sicsú. L.F. de Paulo, orgs, Editora Campus, Rio de janeiro, 1999.

DENIS, Henri. Histoire de la Pensée Economique, Presses Universitaires de France, Paris, 1974.

FRIEDMAN M. "Comments on the Critics", in R.J. Gordon (ed.), Milton Friedman's Monetary Framework: A Debate with His Critics, University of Chicago Press, Chicago, 1974.

HARRIS, Donald. Capital Accumulation and Income Distribution, Standford University Press, Stanford, California, 1978.

HERSCOVICI, Alain. Dinâmica macroeconômica: uma interpretação a partir de Marxe de Keynes, EDUC/EDUFES, São Paulo, 2002.

HERSCOVICI, Alain. Irreversibilidade, Incerteza e Teoria Econômica. Reflexōes a respeito do Indeterminismo Metodológico e de suas Aplicações na Ciência Econômica. Estudos Econômicos (IPE/USP), São Paulo, v. 34, n. 4, p. 805-835, 2004(a).

HERSCOVICI, Alain. Uma crítica do conceito neoclássico de capital: as contribuições de Keynes e de Sraffa, IX Encontro nacional de Economia Política, Uberlândia, 2004(b).

HERSCOVICI, Alain. Historicidade, entropia e não linearidade: algumas aplicaçōes possíveis na Ciência Econômica. Revista de Economia Política, Volume 25, n. 3 (99), julho-setembro 2005.

KAHN, Richard, Selected Essays on employement ad Growth, Cambridge, Cambridge University Press, p. 72-96, 1972.

KEYNES, John Maynard. A teoria geral do emprego, do juro e da moeda, Atlas, São Paulo, 1990

KREGEL, J.A., "Market and institutions as features of a capitalistic production system", Journal of Post-keynesian Economics, Fall 1980, Vol.III, N $1,1980$.

PASINETTI Luigi $L$. "The marginal efficiency of investment, in A "Second Edition" of the General Theory Vol. 1, Edited by G.C. Harcourt and P. a Riach, Rouledge, pp. 185. 197, 1997.

PETRI, Fabio, The "Sraffian" critique of neoclassical economics: some recent developments, Revista da Sociedade Brasileira de Economia Política n. 3, dezembro de 1998, Rio de Janeiro, 1998.

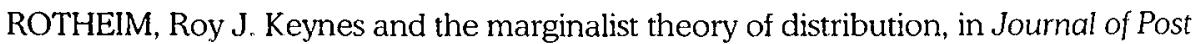
Keynesian Economics/Spring 1988, Vol. 20, N. 3, 1988.

SIMONSEN, Mário Henrique. Dinâmica Macroeconômica, Mc Graw-Hill do Brasil, São Paulo, 1983.

SOLOW, R.M. A Contribution to The theory of Economic Growth", Quartely Journal of Economics, vol. 70, 1956.

VERCELLI Allessandro. Methodological foundations of macroeconomics: Keynes and Lucas, Cambridge Universitry Press, 1991.

VERCELLI Allessandro. Preferência pela liquidez e valor da opção, Economia e Socie dade, Revista do Instituto de Economia da Unicamp, n. 12, Junho /99, Campinas, 1999. 